\title{
Therapeutic Efficacy of Carbon lon Irradiation Enhanced by II-MUA-Capped Gold Nanoparticles: An in vitro and in vivo Study
}

\author{
Pengcheng Zhang ${ }^{1-4}$ \\ Boyi Yu ${ }^{1,2}$ \\ Xiaodong Jin ${ }^{1,2}$ \\ Ting Zhao' \\ Fei $\mathrm{Ye}^{1,2}$ \\ Xiongxiong Liu ${ }^{1,2}$ \\ Ping $\mathrm{Li}^{1,2}$ \\ Xiaogang Zheng' \\ Weiqiang Chen ${ }^{1,2}$ \\ Qiang $\mathrm{Li}^{1,2}$
}

'Institute of Modern Physics, Chinese Academy of Sciences; Key Laboratory of Heavy lon Radiation Biology and Medicine of Chinese Academy of Sciences, Key Laboratory of Basic Research on Heavy lon Radiation Application in Medicine, Lanzhou, Gansu Province, 730000, People's Republic of China; ${ }^{2}$ University of Chinese Academy of Sciences, Beijing, 100049, People's Republic of China; ${ }^{3}$ The First Hospital of Lanzhou University, Lanzhou, 730000 , People's Republic of China; ${ }^{4}$ Department of Radiation Oncology, The First Hospital of Lanzhou University, Lanzhou, 730000, People's Republic of China
Correspondence: Weiqiang Chen; Qiang Li Institute of Modern Physics, Chinese Academy of Sciences; Key Laboratory of Heavy lon Radiation Biology and Medicine of Chinese Academy of Sciences, Key Laboratory of Basic Research on Heavy lon Radiation Application in Medicine, Lanzhou, Gansu Province, 730000, People's Republic of China

Email chenwq73I5@impcas.ac.cn;

liqiang@impcas.ac.cn
Purpose: Gold nanoparticles (AuNPs) are widely studied as radiosensitizers, but their radiosensitization in carbon ion radiotherapy is unsatisfactory. There is a lack of in vivo data on the radiosensitization of AuNPs under carbon ion irradiation. This study focused on the radiosensitization effect of AuNPs in the mouse melanoma cell line B16-F10 in vitro and in vivo.

Materials and Methods: 11-mercaptoundecanoic acid (11-MUA)-coated gold (Au) nanoparticles (mAuNPs) formulations were prepared and characterized. To verify the radiosensitization effect of mAuNPs, hydroxyl radicals were generated in aqueous solution, and the detection of intracellular reactive oxygen species (ROS) and clone survival were carried out in vitro. The tumor growth rate (TGR) and survival of mice were analyzed to verify the radiosensitization effect of mAuNPs in vivo. The apoptosis of tumor cells was detected, and the expression of key proteins in the apoptosis pathway was verified by immunohistochemistry.

Results: The intracellular ROS level in B16-F10 cells was enhanced by mAuNPs under carbon ion irradiation. The sensitization rate of mAuNPs was 1.22 with a $10 \%$ cell survival rate. Compared with irradiation alone, the inhibitory effect of mAuNPs combined with carbon ion irradiation on tumor growth was 1.94-fold higher, the survival time of mice was prolonged by 1.75 -fold, and the number of apoptotic cells was increased by 1.43 -fold. The ratio of key proteins Bax and Bcl2 in the apoptosis pathway was up-regulated, and the expression of caspase-3, a key executor of the apoptosis pathway, was up-regulated.

Conclusion: In in vivo and in vitro experiments, mAuNPs showed radiosensitivity to carbon ion irradiation. The sensitization effect of mAuNPs on mice tumor may be achieved by activating the mitochondrial apoptosis pathway and increasing tumor tissue apoptosis. To our best knowledge, the present study is the first in vivo evidence for radiosensitization of mAuNPs in tumor-bearing mice exposed to carbon ion irradiation.

Keywords: gold nanoparticles, radiosentization, carbon ion irradiation, tumor-bearing mice, apoptosis

\section{Introduction}

Cancer is the second leading cause of death in the world, causing approximately 19.3 million new cases and 9.9 million deaths worldwide in $2020 .^{1}$ Radiotherapy is one of the major cancer treatment modalities as well as surgery and chemotherapy. The purpose of cancer treatment by radiotherapy is to deliver a lethal dose to the tumor while sparing the surrounding normal tissues as much as possible. Compared to conventional radiation such as $\mathrm{X}$ - and $\gamma$-rays, high linear energy transfer (LET) heavy ions exhibit physical and biological advantages like inverted depth-dose distribution, higher relative biological effectiveness (RBE) and low oxygen 
enhancement ratio (OER). ${ }^{2-4}$ Therefore, heavy ion radiotherapy may offer a better option for delivering more doses to the tumor while decreasing radiation damage to normal tissues. ${ }^{5}$ Carbon ion radiotherapy (CIRT) has been utilized in clinical practice for over 25 years to improve therapeutic efficacy. ${ }^{6}$ Although there are several advantages to CIRT, radioresistant tumors, such as malignant mucosal melanoma in the head and neck, it exhibits poor responses even to carbon ion therapy., ${ }^{7,8}$ Undoubtedly, it is still necessary to seek strategies to further improve the therapeutic efficacy in CIRT.

Radiosensitizers can play an important role in enhancing the radiobiological effect. ${ }^{9}$ As a mostly used radiosensitizer, gold $\mathrm{(Au}$ ) nanoparticles (AuNPs) have attracted great interest owing to high X-ray absorption, synthetic versatility, and unique optical, electronic and chemical properties. ${ }^{10,11}$ Multi-disciplinary studies performed over the past decade have demonstrated the potential of AuNPbased radiosensitizers and have identified potential mechanisms underlying the observed radiation enhancement effects of AuNPs since the first in vitro ${ }^{12}$ and in vivo $^{13}$ evidence with X-ray irradiation.

Most efforts in AuNPs-based radiosensitizers were made for conventional radiations such as electron beams, $\mathrm{X}$-rays, and $\gamma$-rays. However, work concerning the radiosensitization of AuNPs under carbon ion irradiation remains rare. Kaur et al found glucose-capped AuNPs in HeLa cells led to a $41 \%$ enhancement of RBE values for carbon ion irradiation. ${ }^{14}$ In a previous study, we found the radio sensitizing effect of AuNPs under carbon ion irradiation was concentration-dependent and the presence of citrate-capped AuNPs achieved sensitizer enhancement ratios of $27 \sim 4 \% \%$ in HeLa cells. ${ }^{15}$ When a reductive drugtirapazamine was conjugated to polyethylene glycolcapped AuNPs, the sensitizer enhancement ratio extended up to $47 \% .{ }^{16}$ Obviously, lack of in vivo studies on the radiosensitizing effect of AuNPs under carbon ion irradiation hampers the utility of AuNPs in carbon ion therapy. In this study, using 11-mercaptoundecanoic acid (11-MUA) coated AuNPs (mAuNPs), we examined the cellular uptake and cytotoxicity of mAuNPs in a murine melanoma cell line, and the radiosensitizing effect of mAuNPs in vitro. More importantly, tumor-bearing mice were utilized to determine the radiosensitizing effect of mAuNPs under carbon ion irradiation and possible mechanism underlying the in vivo radiosensitization of mAuNPs was analyzed.

\section{Materials and Methods}

\section{Synthesis and Characterization of} mAuNPs

We produced 11-mercaptoundecanoic acid-capped AuNPs (mAuNPs) using a ligand exchange method starting from citrate-capped AuNPs (Supporting Information). ${ }^{17,18}$ The as-synthesized mAuNPs were characterized by optical absorption spectrum, transmission electron microscopy (TEM), dynamic light scattering (DLS) and inductively coupled plasma atomic emission spectrometer (ICP-AES). Absorption spectra were measured using a microplate reader (Multiskan spectrum 1500, Thermo Electron Co., Finland). The TEM measurements were performed using a Tecnai TF20 transmission electron microscopy (FEI Co., USA) at $200 \mathrm{kV}$. The concentration of AuNPs was measured using an IRIS ER/S ICP-AES (TJA Co., USA) after the AuNPs had been dissolved by aqua regia. The hydrodynamic diameter and zeta potential of AuNPs were measured at room temperature by dynamic light scattering using a zeta particle size analyzer (Nano-ZS, Malvern, England). The data were collected on an autocorrelator with a detection angle of scattered light of $173^{\circ}$.

\section{Cell Culture}

The murine melanoma cell line B16-F10 was purchased from the Cell Bank of the Chinese Academy of Sciences (Shanghai, China) and was preserved in our laboratory. B16-F10 cells were cultured in a Dulbecco's modified Eagle's complete medium (DMEM) (Hyclone, US) supplemented with $10 \%$ fetal bovine serum (FBS) (Hyclone, US), $100 \mathrm{U} / \mathrm{mL}$ penicillin, and $100 \mu \mathrm{g} / \mathrm{mL}$ streptomycin (Hyclone, US). Cell cultures were kept at $37^{\circ} \mathrm{C}$ in a $5 \%$ $\mathrm{CO}_{2}$ humidified incubator.

\section{Cellular Uptake of mAuNPs}

The cells were co-cultured with AuNPs-containing medium at Au concentrations of 5.0, 10.0, 20.0, 40.0, 60.0 $\mu \mathrm{g} /$ $\mathrm{mL}$ for $24 \mathrm{~h}$, respectively, and the medium was discarded. B16-F10 cells were then washed with PBS and harvested using trypsin (Hyclone, US). The cell number was counted using a particle counter (Z1, Beckman Coulter Co., US). The cell suspension was centrifuged for $20 \mathrm{~min}$ at $12,000 \mathrm{rpm}$. The collected cells are ruptured using ultrapure water using osmotic pressure. The cells are also broken more thoroughly with ultrasound to ensure that the Au inside the cells is completely released. The pellets were dissolved by aqua regia, and then the solution was 
diluted with ultrapure water. The concentration of $\mathrm{Au}$ was determined using ICP-AES, and the results were normalized by cell numbers. The concentration of mAuNPs used in all subsequent cell experiments was the same as that used in the co-culture concentrations.

\section{Location and Measurement of mAuNPs Taken Up by Cells}

The location of the mAuNPs captured by cells was determined with biological transmission electron microscopy and dark field microscopy. After a 24-hour incubation with mAuNPs at a concentration equivalent to single cell uptake, trypsinized B16-F10 cells were harvested by centrifugation and fixed overnight at $4^{\circ} \mathrm{C}$ in $3 \%$ glutaraldehyde solution (in PBS). The fixed cells were then dehydrated, polymerized, sectioned, and finally doublestained with uranyl acetate and lead citrate. Observations were performed on a JEM-1230 transmission electron microscopy (JEOL Co., Japan) at $100 \mathrm{kV}$. For the dark field microscopy experiment, B16-F10 cells were cultured with AuNPs for 24 hours in a $\Phi 35 \mathrm{~mm}$ Petri dish with a coverslip placed in advance. The coverslip was placed on a glass slide and then observed under a dark field microscope (Olympus, Japan).

\section{Cell Viability}

The effects of AuNPs on cell activity were assayed using a CCK-8 kit (Beyotime, China). Briefly, cells were grown in 96-well plates with 2000 cells per well and were cocultured for $24 \mathrm{~h}$ with medium containing mAuNPs at $\mathrm{Au}$ concentrations of $0.1,1.0,2.0,5.0,10.0,20.0$, and $40.0 \mu \mathrm{g} /$ $\mathrm{mL}$, respectively. The medium containing mAuNPs was discarded and co-incubated with medium containing CCK8 reagent again for $1.5 \mathrm{~h}$ and then assayed using a microplate reader with a wavelength of $450 \mathrm{~nm}$.

\section{Irradiation}

For the preparation of aqueous solutions and cell exposure treatments, samples were set horizontally to be irradiated with a carbon ion beam with initial energy of $190 \mathrm{MeV} / \mathrm{u}$ at the Heavy Ion Therapy Center in Wuwei, China. For aqueous solutions and in vitro studies, the energy of the carbon ion beam was adjusted through degraders so as to provide an LET value of $50 \mathrm{keV} / \mu \mathrm{m}$ on samples and the dose rate was set at $0.4 \mathrm{~Gy} / \mathrm{min}$. For in vivo experiments, a carbon ion beam of $260 \mathrm{MeV} / \mathrm{u}$ was supplied, and a range filter was used to build a spread-out Bragg peak (SOBP) of 6-cm width for the irradiation of tumor-bearing mice at 4.0 Gy $\mathrm{RBE}$ at a dose rate of $0.3 \mathrm{~Gy} / \mathrm{min}$. Each mouse was placed into a $50 \mathrm{~mL}$ conical tube for immobilization. The position of the tumor tissue of each mouse was aligned to the hole of a lead plate for irradiation with carbon ions.

X-ray irradiation was performed with an X-RAD 320 X-ray apparatus (Precision Co., Ltd., USA) operated at $225 \mathrm{kV}$ and $13.3 \mathrm{~mA}$. The dose rate was $1.96 \mathrm{~Gy} / \mathrm{min}$ for $\mathrm{X}$-ray irradiation, and total radiation dose was controlled through the irradiation time.

Irradiation was performed at room temperature, and the control group was sham-irradiated.

\section{Clonogenic Survival Assay}

All treatments were carried out in quadruplicate in $\Phi 60-\mathrm{mm}$ Petri dishes. Briefly, B16-F10 cells irradiated with carbon ions at doses of $0,1.0,2.0,3.0,4.0$, and 6.0 Gy were trypsinized, harvested, counted using the Z1 Coulter particle counter, diluted, and then seeded into $\Phi 60-\mathrm{mm}$ Petri dishes with $5 \mathrm{~mL}$ complete media. After incubation for a week, cells were stained with crystal violet for $30 \mathrm{~min}$ and then carefully washed. Colonies with more than 50 cells were recorded as surviving cells and were counted manually under an inverted microscope. Cell survival data were fitted using the linearquadratic (LQ) model, and the sensitizer enhancement ratio (SER) of mAuNPs was calculated at the 10\% survival level.

\section{Measurement of Hydroxyl Radical and Reactive Oxidative Species}

Hydroxyl radical production was evaluated using 3-coumarin carboxylic acid (3-CCA, J\&K Chemical Co. Ltd, China) as a probe. The 3-CCA solution was prepared following the reported procedure, ${ }^{19}$ The diluted solutions, with mAuNPs concentrations of $0,1.0,5.0,10.0,20.0$, and $30.0 \mu \mathrm{g} / \mathrm{mL}$, were placed in $\Phi 35-\mathrm{mm}$ Petri dishes, and irradiated at 2 Gy according to the irradiation condition. After irradiation, the solutions were equally dispensed into wells of 96-well black plates, and measurements were taken at an excitation wavelength of $395 \mathrm{~nm}$ and an emission wavelength of $442 \mathrm{~nm}$ with a microplate reader (Infinite F200/M200, TECAN Co., Switzerland). The entire procedure was protected from light.

Reactive oxidative species (ROS) production was measured using the DCFH-DA assay, which is a fluorogen dye measuring hydroxyl, peroxyl, and other ROS activities within cells. B16-F10 cells were grown on glass 
coverslips in cell culture dishes. After co-cultivation with mAuNPs, the Au-containing medium was discarded. Cells were then co-cultured with DCFH-DA solution, which was diluted in serum-free medium for 20 minutes before irradiation. After irradiation at $2.0 \mathrm{~Gy}$, the coverslips were placed on ice and quickly photographed with a fluorescence microscope. All the samples were photographed with the same microscopic parameters. The fluorescent intensities of cells were analyzed using ImageJ software. Finally, the fluorescence intensity per unit area under each treatment was calculated to ensure reliable results; at least 200 cells were randomly counted for each sample. Results are expressed as mean \pm standard deviation.

\section{Establishment of Tumor-Bearing Mice and Treatment with AuNPs}

All animal experiments were performed according to the National Institutes of Health guidelines and Animal Use Protocol at the Institute of Modern Physics, and were approved by the Institutional Animal Care and Use Committee (IACUC) at the Institute of Modern Physics. C57/BL6 mice (females, $\sim 16-17 \mathrm{~g}$ weight at the time of purchase), obtained from the Lanzhou Veterinary Research Institute of the Chinese Academy of Agricultural Sciences (Lanzhou, China), were used in the in vivo study. Before the experiment, the mice were acclimatized for one week. The temperature of the feeding environment was set at $26^{\circ} \mathrm{C}$ with normal circadian rhythm and free access to food and water.

B16-F10 cells were employed to establish tumorbearing mice. Cells in the logarithmic growth phase were collected and resuspended with saline to $10^{7} / \mathrm{mL}$ after digestion with trypsin. A xenograft tumor was formed by subcutaneously injecting $100 \mu \mathrm{L}\left(10^{6}\right.$ cells $)$ of the cell suspension into the right hind leg of the mouse. The tumor volume of mice reached approximately $100 \mathrm{~m}^{3}$. The long diameter (L) and short diameter (W) of the tumor were measured, and the tumor volume $(\mathrm{V})$ was calculated using the formula: $\mathrm{V}=0.5 \times \mathrm{L} \times \mathrm{W}^{2,20}$

The tumor-bearing mice were randomly divided into four groups: (1) control ( $\mathrm{n}=9$ ); (2) mAuNPs injection only (mAuNPs) ( $\mathrm{n}=9)$; (3) carbon ion irradiation alone $(\mathrm{n}=9)$ (CI); and (4) combination of mAuNPs injection with carbon ion irradiation (mAuNPs-CI) $(n=9)$.

Intratumoral injection was selected for treatment with AuNPs to the tumor, $30 \mathrm{~min}$ before irradiation. The amount of injected mAuNPs per mouse was $100 \mu \mathrm{L}$ with a concentration of $2.8 \mathrm{mg} / \mathrm{mL}$ in Au. After irradiation, the long and short diameters of the tumors in mice were measured every other day, and the tumor volume $V_{t}$ at the day when measurement was taken was calculated according to the above-mentioned formula. The tumor volume measured before irradiation was $\mathrm{V}_{0}$, then the tumor growth rate (TGR) was calculated according to the formula: $\mathrm{TGR}=\mathrm{V}_{\mathrm{t}} / \mathrm{V}_{0}$.

The survival status of the mice in each group was recorded until their death.

\section{Mouse Pathological Sections and Tunel Experiment}

Three mice were randomly selected from each group, euthanized 24 hours after irradiation, and the tumor tissue was immediately excised and then fixed in paraformaldehyde for 24 hours. The tumor tissues were embedded in paraffin, sliced and dewaxed. Five sections of each tumor tissue were subjected to HE staining and image acquisition. Another five sections of each tumor tissue were tested to determine the number of apoptotic cells in the tumor tissue using the Apoptosis Detection Kit (Roche, Switzerland). Sections were observed with a microscope and photographed.

\section{immunohistochemistry}

Immunohistochemical studies were performed to detect the expression of $\mathrm{Bax}, \mathrm{Bcl} 2$, and caspase- 3 proteins on tumor sections. Briefly, the sections were examined under a light microscope and photographed after dewaxing and hydration, antigen retrieval, blocking, incubation with primary and HRP-coupled secondary antibodies, diaminobenzidine (DAB) development, and hematoxylin counterstaining, respectively. The above reagents were purchased from Wuhan Sanying Biotechnology Company. Protein expression was quantified using Image J (National Institutes of Health, USA).

\section{Statistics}

Each experiment was performed in triplicate and data presented in representation of individual experiments. A two-tailed Student's $t$-test and one-way analysis of variance (ANOVA) were used to evaluate the statistical significance between different groups. Statistical analyses were performed with the SPSS 19.0 software. P-values $<0.05$ were considered significantly different. 


\section{Results}

\section{Synthesis and Characterization of mAuNPs}

First citrate-capped AuNPs (cAuNPs) with a size of 14.45 $\pm 1.99 \mathrm{~nm}$ were synthesized using a previously reported procedure (Figure 1A and B). ${ }^{15}$ After ligand exchange from citrate to 11-MUA, the size of 11-MUA capped AuNPs (ie, mAuNPs) had a mean diameter of $15.97 \pm 1.35 \mathrm{~nm}$, which was slightly larger than that of cAuNPs (Figure 1C and D). The hydrodynamic diameter of cAuNPs was $30.64 \mathrm{~nm}$ with a Zeta potential of $-18.1 \mathrm{mV}$, which is in line with the values reported previously. ${ }^{21,22}$ After the ligand exchange, the hydrodynamic diameter of mAuNPs increased to $32.87 \mathrm{~nm}$. Since the surface of mAuNPs was covered by 11-MUA, the outside of mAuNPs was still surrounded by carboxylic acid and the Zeta potential changed to $-15.6 \mathrm{mV}$ (Figure 1E). The surface plasmon resonance absorption of AuNPs occurs at $520 \mathrm{~nm},{ }^{23}$ regardless of how the surface of the AuNPs is modified. When mAuNPs were suspended in culture medium, the characteristic peak of AuNPs red-shifted to $530 \mathrm{~nm}$ without any broadening even after 24 hours (Figure S1). No significant changes in the dispersion and particle size of mAuNPs were observed by TEM after a 24-hour co-culture with the medium (Figure $\underline{\mathrm{S} 2}$ ). Collectively, mAuNPs were prepared with high stability. Our results coincided with those of previous reports. ${ }^{24}$

\section{Cellular Uptake of mAuNPs}

Shown in Figure 2A are photographs taken under dark field microscope of B16-F10 cells cultured with and without mAuNPs for $24 \mathrm{~h}$. Numerous small spots in B16-F10 cells co-cultured with mAuNPs for $24 \mathrm{~h}$ were visible compared to controls, indicating that mAuNPs entered B16-F10 cells. Next, we investigated the cellular uptake of mAuNPs after B16-F10 cells were co-cultured with mAuNPs at different concentrations by ICP-AES (Figure 2B). The amount of cellular uptake of mAuNPs increased with the AuNPs co-culture concentrations in the range of $\sim 0-60 \mu \mathrm{g} / \mathrm{mL}$. The cellular uptake amount reached $3.6 \mathrm{pg} / \mathrm{cell}$ at the co-culture concentration of 10 $\mu \mathrm{g} / \mathrm{mL}$. If the weight of $10^{9}$ cells was approximately $1 \mathrm{~g}$, the average intracellular concentration of mAuNPs was about 360-fold higher than the concentration of mAuNPs co-cultured with B16-F10 cells. Compared with the cAuNPs, the intracellular uptake of mAuNPs was also higher (Figure S3). TEM images indicated that mAuNPs were gathered into vesicles in the cytoplasm (Figure 2C). These results are consistent with those in the previous study. $^{15}$

\section{Cytotoxicity and Clonogenic Survival Assay}

Since B16-F10 cells exhibited high uptake of mAuNPs, we further studied the cytotoxicity of mAuNPs. Cell viability in the presence of mAuNPs at various concentrations is shown in Figure 3A. Clearly, the toxicity of mAuNPs to B16-F10 cells was low, because the cell viability was higher than $80 \%$ when the concentration of mAuNPs reached $10 \mu \mathrm{g} / \mathrm{mL}$. In our previous study, ${ }^{15}$ we found that the radiosensitizing effect of cAuNPs was concentration-dependent. In this study, we selected $6 \mu \mathrm{g} / \mathrm{mL}$ as the co-culture concentration of mAuNPs







Figure I Characterization data for mAuNPs. Transmission electron micrographs and size distributions of the as-synthesized cAuNPs (A, B) and mAuNPs (C, D), (E) Hydrodynamic diameters and Zeta potentials of cAuNPs and mAuNPs measured with dynamic light scattering (DLS). Note: hydrodynamic diameter and Zeta potential measurements are presented in the supporting information. 




B

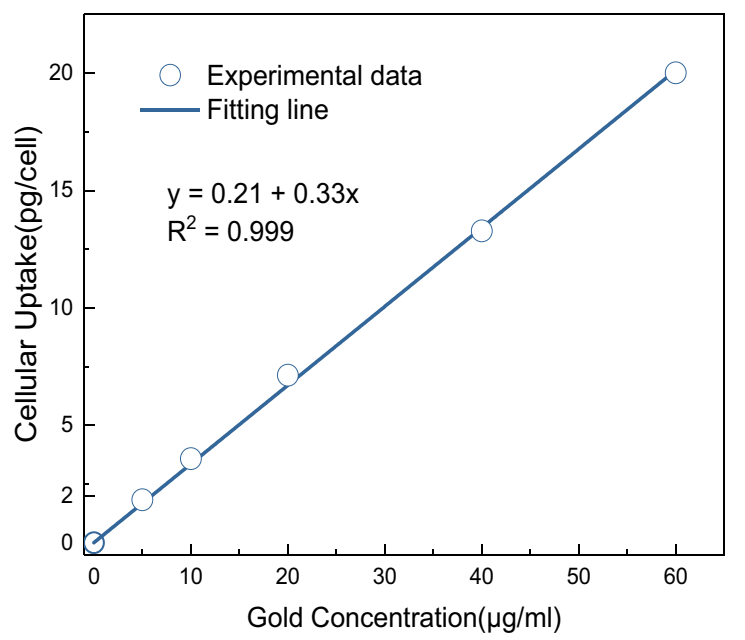

C



Figure 2 Uptake and distribution of mAuNPs in cells. (A) Photographs taken under dark field microscope for BI6-FI0 cells cultured with or without mAuNPs for 24h, The cell nuclei were dyed with Hoechst 33342. The yellow arrow shows the autofluorescence of mAuNPs observed in the dark-field microscope. The scale bars represent $10 \mu \mathrm{m}$. (B) Uptake of mAuNPs by BI6-FIO cells at various co-culture concentrations. (C) Transmission electron micrographs of BI6-FIO cells cultured with and without mAuNPs. The scale bars (left and right) represent $5 \mu \mathrm{m}$ and $500 \mathrm{~nm}$, respectively.

for the subsequent clonogenic survival experiments. As shown in Figure 3B, the combination of carbon ion irradiation with mAuNPs led to decrement in survival fraction than carbon ion irradiation alone. Relative biological effectiveness (RBE) values were calculated from the fitted line of the experimental data using the linear quadratic (LQ) model at $10 \%$ survival level. ${ }^{25,26}$ The RBE value of the combined treatment of mAuNPs plus carbon ion irradiation) was 1.44, and increased by a factor of 1.22 compared to that of carbon ion irradiation alone (Table 1). The SER value of mAuNPs therefore was 1.22. Thus, the results demonstrated that mAuNPs enhanced the damage induced by carbon ion irradiation of B16-F10 cells.

\section{In vivo Study}

The tumor growth rates of tumor-bearing mice under the different treatments are shown in Figure 4A. There was no significant difference between the control and mAuNPstreated groups, which is consistent with the low cytotoxicity of mAuNPs obtained above. The tumor growth rates reached approximately 15.2-fold after 14 days for the control and in mAuNPs-treated groups. The tumorbearing mice treated with carbon ion irradiation alone showed an obvious inhibition in tumor growth after 4 days post-irradiation, corresponding to a tumor growth rate of 9.7-fold. The combined treatment of mAuNPs and carbon ion irradiation led to a much smaller tumor growth 
A



B



Figure 3 Cell viability of gold nanoparticles and the radiation enhancement in cells. (A) Viability of BI6-FI0 cells in the presence of mAuNPs at various concentrations. (B) Survival curves of BI6-FIO cells with or without mAuNPs in co-culture (mAuNPs concentration of $6 \mu \mathrm{g} / \mathrm{mL}$ ) after carbon ion irradiation. The survival curve of BI6-FI0 cells exposed to X-rays is shown in order to calculate the RBE values under the conditions of carbon ion irradiation with and without mAuNPs.

rate in the first 8 days and the tumor growth rate of the combined treatment was only 4.6 after 14 days postirradiation (Figure 4A).

The Kaplan-Meier survival curves of all the groups are shown in Figure 4B. Compared to the control (treated with intratumoral injection of PBS), the administration of mAuNPs did not elicit any increment in survival of tumorbearing mice. All mice died 16 days after treatment. Carbon ion irradiation significantly prolonged the survival of tumor-bearing mice to 24 days, thereby leading to an improvement of $50 \%$ in contrast with the control and AuNPs-treated groups. The survival of mice in the combined treatment group extended to 42 days, and was about $75 \%$ longer than that of mice irradiated with carbon ions alone. The survival of mice with the combined treatment was significantly prolonged compared to the mice in the control, mAuNPs-treated only, and irradiation alone groups ( $\mathrm{P}<0.05$; Log rank test). Thus, our in vivo results definitely indicated that mAuNPs enhanced the therapeutic effects of carbon ion irradiation on mice tumors, results which were in line with the clonogenic survival study in vitro. Collectively, the in vivo and in vitro studies provided strong evidence that the application of mAuNPs improved the therapeutic efficacy of carbon ions.

Pathology analysis of the tumor tissues resected from the mice in the different treatment groups was conducted. As shown in Figure 5A, there were no significant damages in the tumor tissues of the mice in the control and mAuNPs alone groups. Tumor tissue presented obvious necrosis and bleeding after the combined treatment with mAuNPs and carbon ion irradiation, and the nuclei of the necrotic tumor cells condensed and ruptured. Similar phenomena were also observed in the irradiation alone group, but the degree of the pathological changes decreased.

Evidence of apoptosis, a determinant of radiosensitivity ${ }^{27,28}$ was also observed in cancer cells exposed to carbon ion irradiation. ${ }^{29}$ Therein, we examined whether apoptosis was associated with the radiosensitizing effect of mAuNPs through in vivo study under carbon ion irradiation. As shown in Figure 5B and C, after irradiation, the number of apoptotic cells observed in the Tunel-stained sections was 10.5-fold higher than that in the control samples. Notably, the

Table I Radiobiological Parameters for BI6-FI0 Cells Irradiated with Carbon lons $(50 \mu \mathrm{m} / \mathrm{keV})$ in the Presence of mAuNPs

\begin{tabular}{|l|c|c|c|c|c|}
\hline & $\boldsymbol{\alpha}\left(\mathbf{G y}^{-1}\right)$ & $\boldsymbol{\beta}\left(\mathbf{G y} \mathbf{~}^{-\mathbf{2}}\right)$ & $\mathbf{D}_{\mathbf{1 0}}(\mathbf{G y})$ & SER & RBE \\
\hline X-rays & $0.121 \pm 0.018$ & 0.08 & 4.69 & - & - \\
Carbon ions alone & $0.258 \pm 0.022$ & 0.08 & 3.97 & - & 1.18 \\
mAuNPs + carbon ions & $0.443 \pm 0.041$ & 0.08 & 3.26 & 1.22 & 1.44 \\
\hline
\end{tabular}

Abbreviations: $D_{10}$, the dose required to decrease the survival fraction to $10 \%$; SER, sensitizer enhancement ratio; RBE, relative biological effectiveness. 



Figure 4 Effects of AuNPs on tumor size and survival in mice. (A) Tumor growth rates of the mice in the different groups; (B) Survival of the tumor-bearing mice in the different groups $(*, p<0.05 ; * *, p<0.01)$.

number of apoptotic cells detected in the combined treatment group increased by a factor of 1.8 -fold compared to the irradiation alone group (Figure 5C). These results demonstrated that the administration of mAuNPs greatly enhanced the cellular apoptosis induced by carbon ion irradiation. There was a synergistic effect of mAuNPs and carbon ion irradiation, contributing to the improvement of the therapeutic efficacy of carbon ions.

The immunohistochemistry studies revealed brownyellow staining (Figure 6A) to indicate the positive expression of the corresponding protein. We used Image $\mathrm{J}$ software to quantify the optical density values of the tan-positive expression fraction and quantify the expression of each protein. Figure 6B reports the ratio of Bax expression to $\mathrm{Bcl} 2$ expression $(\mathrm{Bax} / \mathrm{Bcl} 2$ ratio) in the tumor tissue of mice treated with mAuNPs, which increased significantly compared to the control group. The quantitative analysis of Caspase- 3 expression in mouse tumors indicated that Caspase- 3 expression was upregulated in all tumors of mice irradiated with Carbon ion irradiation, and Caspase-3 expression was significantly upregulated in tumor tissues of mice treated with mAuNPs compared to controls (Figure 6C).

\section{Discussion}

\section{Radiosensitizing Mechanism of mAuNPs Under Carbon Ion Irradiation}

According to different time scales, the interaction between organisms and ionizing radiation will go through three stages: physical, chemical, and biological. ${ }^{30}$ The main mechanism of AuNPs radiosensitizer activity is based on differences in energy absorption characteristics between $\mathrm{Au}$ and surrounding tissue. ${ }^{11}$ Differences in energy absorption characteristics of tissues result in an enhanced absorption of the physical dose in the presence of Au. However, by Monte Carlo simulation, Rudek et al pointed out that the enhancement of physical dose under carbon ion irradiation cannot be ignored. ${ }^{31}$ Fuss et al also excluded the importance of any indirect physical effects, that is, the dose enhancement only depends on the interaction between secondary electrons and AuNPs. ${ }^{32}$

Carbon ion irradiation led to the targeted necrosis and apoptosis of tumor tissue. The addition of mAuNPs could enhance the number of apoptotic cells in the tumor tissue. Apoptosis induced by carbon ion irradiation originates from the increased ROS in cells. ${ }^{33,34}$ The indirect action of hydroxyl radicals in low LET irradiation was responsible for about $70 \%$ of the cell killing effect. In high LET carbon ion beam irradiation, the killing effect of hydroxyl radicals was not as pronounced as in low LET irradiation, but it also contributed to about $30 \%$ of activity. ${ }^{35,36} \mathrm{We}$ examined the production of hydroxyl radicals in aqueous solution with or without the presence of mAuNPs under carbon ion irradiation. As shown in Figure 7A and B, the radiation enhancement ratio reached 2.8 in the presence of $20 \mu \mathrm{g} / \mathrm{mL}$ mAuNPs under carbon ion irradiation. This is consistent with our previous study using cAuNPs. ${ }^{15}$ Furthermore, the intracellular ROS levels of B16-F10 cells treated differently were investigated using DHFA as 



Figure 5 Mouse tumor tissue sections and Tunel experiment. (A) Images of tumor tissue sections stained with HE for the mice in the different groups. Tumor tissue showing obvious necrosis (yellow arrow) 24-h post-irradiation, with the extent of necrotic tissue increasing significantly following the addition of mAuNPs. The scale bars represent $100 \mu \mathrm{m}$; (B) Apoptotic cells in tumor tissues detected with the Tunel method $24 \mathrm{~h}$ after treatment. The scale bars represent $50 \mu \mathrm{m}$; (C) Quantitative analysis of apoptosis. The number of apoptotic cells in the tumor tissues was calculated by averaging the number of positive Tunel signals from eight fields with highest density of Tunel signals in each section. $(* *, p<0.01)$. 



Figure 6 Immunohistochemistry images and analysis results. (A) Photographs of immunohistochemistry. DAB color development, brown-yellow staining is positive cells. The scale bars represent $50 \mu \mathrm{m}$; (B) The ratio of Bax expression to Bcl2 expression; (C) Expression analysis of active caspase 3. (**, $<<0.01$ ).

probes (Figure 7C). ${ }^{37}$ The ROS level in cells treated with the combination of mAuNPs and carbon ion irradiation was 1.43-fold higher than that in the irradiated cells alone without NPs (Figure 7D). Arguably, the increased apoptosis induced by adding mAuNPs to tumor tissue might be attributed to the increment of hydroxyl radical and ROS levels. Several recent studies ${ }^{38-40}$ have also reported that the presence of ROS increased the toxicity of cells, which further validates our results.

DNA in cancer cells has always been considered as the radiation target. Since the presence of $\mathrm{mAuNPs}$ decreased the survival of B16-F10 cells under carbon ion irradiation, mAuNPs could potentially enhance DNA damage in cancer cells exposed to carbon ions. We measured $\gamma$-H2AX foci, a marker of DNA double-strand breaks (DSBs), ${ }^{41}$ in B16-F10 cells cultured with and without mAuNPs after irradiation with carbon ions. There was no significant difference in the number of $\gamma-\mathrm{H} 2 \mathrm{AX}$ foci between the combined treatment and exposure to irradiation alone (Figure S4). Additionally, the cellular location of mAuNPs captured by cells was verified to be in the cytoplasm instead of the nuclei in this study. Thus, the sensitizing activity of mAuNPs to carbon ion irradiation might originate from the cytoplasm rather than in the nuclei.

We proposed that mAuNPs-enhanced hydroxyl radical and ROS levels in the cytoplasm might led to further damage to mitochondria. Thus, we examined the expression of key proteins on the apoptotic pathway (Bax, Bcl2, and Caspase-3) using immunohistochemistry assays. The formation of heterodimers in the Bcl-2 family of pro-apoptotic and anti-apoptotic proteins can turn apoptosis on or off as the heterodimer is responsible for mutual neutralization of the bound pro- 
A


B


Figure 7 Enhanced radiation in solution and increased production of intracellular ROS. (A) Fluorescence intensity varies dependent on the dose of carbon ion irradiation; (B) Enhancement ratio of hydroxyl radical production in aqueous solution of AuNPs at different concentrations under carbon ion irradiation; (C) Fluorescent images of ROS with DCHF-DA in BI6-FIO cells after irradiation at 2 Gy in the presence of mAuNPs. The scale bars represent $50 \mu \mathrm{m}$; (D) The fluorescence intensity per unit area in BI6FIO cells after irradiation. (*, $\mathrm{p}<0.05, * *, p<0.0 \mathrm{I})$.

apoptotic and anti-apoptotic proteins. Thus, the balance between the expression levels of protein units (eg, Bcl-2 and Bax) is critical for cell survival or death; more specifically, upregulation of the $\mathrm{Bax} / \mathrm{Bc} 12$ ratio predicts an increase in tissue apoptosis. ${ }^{42,43}$ Upregulation of Caspase-3 expression was also observed. Caspase-3 is a key executioner in signaling pathways leading to apoptosis. ${ }^{44,45}$ Our observation at the cellular level demonstrated that cells treated with AuNPs exhibited a significant increase in intracellular ROS after irradiation, which promotes apoptosis. Consequently, the mitochondrial apoptosis pathway is activated and then the apoptotic rate of cells increases, ${ }^{46-48}$ thereby leading to the radiosensitization of mAuNPs to carbon ion irradiation. Nevertheless, the underlying mechanisms responsible for the radiosensitizing effect of $\mathrm{mAuNPs}$ remain to be investigated in a future study.

\section{Surface Modification or Ligand Effect of AuNPs}

It is well known that surface structure modification of AuNPs greatly influences their sensitization to ionizing radiation, as well as their size and morphology. ${ }^{49}$ In the study by Kaur et al, thioglucose-capped AuNPs with a size of $15 \mathrm{~nm}$ used in HeLa cells led to an enhancement of $41 \%$ of the RBE value of carbon ions. Our previous work showed that cAuNPs enhanced carbon ion-induced damages to HeLa cells by $\sim 27-44 \% .{ }^{15}$ Thus, in the present study, we selected AuNPs with a size of $15 \mathrm{~nm}$ and modified the surface of AuNPs with 11-MUA, which resulted in an increased cellular uptake of mAuNPs compared to cAuNPs. Obviously, the surface modification, or so-called ligand effect, of AuNPs played an important role 
in sensitizing cancerous cells or tumor tissues to carbon ions. As it is possible for mAuNPs to be modified further due to the presence of the carboxylic acid residue of 11MUA, mAuNPs might be an excellent candidate for further studies.

\section{Administration of AuNPs}

The radiosensitizing effect of AuNPs depends not only on their physical and chemical properties, but also on the administration strategy, the type of ionizing radiation, and the corresponding radiation dose. Chang et al observed significant suppression of tumor growth with statistical survival benefits by the intravenous administration of AuNPs $(672 \mu \mathrm{g})$ combined with a single fraction of $6 \mathrm{MeV}$ electron beam at $25 \mathrm{~Gy} .{ }^{50}$ Anijdan et al found that tumor growth was partially inhibited, albeit without a statistically significant survival benefit, following intratumoral injection of AuNPs ( 500-1000 $\mu \mathrm{g})$ under X-ray irradiation at $20 \mathrm{~Gy}^{51}$ The study by Komatsu et $\mathrm{al}^{52}$ demonstrated regardless of intravenous administration or intratumoral injection of AuNPs $(400 \mu \mathrm{g})$, X-ray irradiation at 10 Gy showed no tumor inhibition or statistical survival benefit. In our study, intratumoral injection of mAuNPs $(280 \mu \mathrm{g})$ combined with carbon ion irradiation at 4 Gy contributed to a significant radiation enhancement of $94 \%$ in tumor growth inhibition and a significant $75 \%$ improvement in survival time. However, the location and distribution of mAuNPs in the tumor tissues remained unknown. To make full use of the radiosensitizing action of mAuNPs and to clarify the corresponding mechanisms underlying the radiosensitization of mAuNPs, the distribution of mAuNPs in the tumor tissues after intratumoral injection should be examined in a future study. Arguably, intravenous administration of AuNPs is a more favorable approach for clinical application. In any event, administration of AuNPs for radiosensitization remains to be investigated comprehensively.

\section{Conclusion}

The as-synthesized mAuNPs exhibited low cytotoxicity and high cellular uptake in melanoma B16-F10 cells under investigation. The presence of mAuNPs led not only to a reduction of cell survival and an increase in RBE values but also significantly suppressed tumor growth and prolonged tumor-bearing mice survival under carbon ion irradiation. mAuNPs effectively induced a radiosensitizing effect in in vitro cells and in vivo tumors exposed to carbon ions. Thus, our findings provide evidence that mAuNPs improve the therapeutic efficacy of carbon ion therapy. The increased rate of apoptosis in mouse tumor tissues may be due to the production of hydroxyl radicals in the presence of mAuNPs and the increased levels of ROS, which in turn activate the mitochondrial apoptotic pathway. Our study sheds light on the potential clinical applications of mAuNPs in carbon ion therapy.

\section{Abbreviations}

AuNPs, gold nanoparticles; mAuNPs, 11mercaptoundecanoic acid coated gold nanoparticles; 11MUA, 11-mercaptoundecanoic acid; SER, sensitizer enhancement ratio; ROS, reactive oxygen species; TEM, Transmission electron microscope; DLS, Dynamic light scattering; LET, linear energy transfer; RBE, relative biological effectiveness; OER, oxygen enhancement ratio; CIRT, Carbon ion radiotherapy; SOBP, spread-out Bragg peak; DCFH-DA, 2',7'dichlorofluorescein diacetate; TUNEL, terminal deoxynucleotidyl transferase-mediated dUTP nick-end labeling; cAuNPs, citrate-coated AuNPs.

\section{Ethics Approval}

All animal experiments were approved by the Academic Committee of the Institute of Modern Physics, Chinese Academy of Sciences (No. 2018(006)). All animal experiments were performed in the SPF class animal house at the Institute of Modern Physics, Chinese Academy of Sciences.

\section{Funding}

This work is jointly supported by the Key Deployment Project of Chinese Academy of Sciences (Grant No. KFZD-SW-222), the National Natural Science Foundation of China (Grant No. 11875299), the Key R\&D Program of Gansu Provincial Department of Science and Technology (Grant No. 20YF3FA028), Gansu Health Industry Scientific Research Project (Grant No. GSWSKY-2019-79), In-hospital Funded Project of Lanzhou University First Hospital (Grant No. ldyyyn 2018-21), the Science and Technology Project of Chengguan District, Lanzhou City (Grant No. 20202-2-6).

\section{Disclosure}

The authors report no conflicts of interest in this work. 


\section{References}

1. Sung H, Ferlay J, Siegel RL, et al. Global cancer statistics 2020 : GLOBOCAN estimates of incidence and mortality worldwide for 36 cancers in 185 countries. CA Cancer J Clin. 2021;71(3):209-249. doi: $10.3322 /$ caac. 21660

2. Loeffler JS, Durante M. Charged particle therapy-optimization, challenges and future directions. Nat Rev Clin Oncol. 2013;10 (7):411-424. doi:10.1038/nrclinonc.2013.79

3. Durante M, Debus J. Heavy charged particles: does improved precision and higher biological effectiveness translate to better outcome in patients? Semin Radiat Oncol. 2018;28(2):160-167. doi:10.1016/j. semradonc.2017.11.004

4. Tinganelli W, Durante M, Hirayama R, et al. Kill-painting of hypoxic tumours in charged particle therapy. Sci Rep. 2015;5(1):17016. doi:10.1038/srep17016

5. Halperin EC. Particle therapy and treatment of cancer. Lancet Oncol. 2006;7(8):676-685. doi:10.1016/S1470-2045(06)70795-1

6. Durante M, Orecchia R, Loeffler JS. Charged-particle therapy in cancer: clinical uses and future perspectives. Nat Rev Clin Oncol. 2017;14(8):483-495. doi:10.1038/nrclinonc.2017.30

7. Yanagi T, Mizoe J, Hasegawa A, et al. Mucosal malignant melanoma of the head and neck treated by carbon ion radiotherapy. Int J Radiat Oncol Biol Phys. 2009;74(1):15-20. doi:10.1016/j.ijrobp.2008.07.056

8. Mizoe JE, Hasegawa A, Jingu K, et al. Results of carbon ion radiotherapy for head and neck cancer. Radiother Oncol. 2012;103 (1):32-37. doi:10.1016/j.radonc.2011.12.013

9. Begg AC, Stewart FA, Vens C. Strategies to improve radiotherapy with targeted drugs. Nat Rev Cancer. 2011;11(4):239-253. doi:10.1038/nrc3007

10. Liu Y, Zhang P, Li F, et al. Metal-based NanoEnhancers for future radiotherapy: radiosensitizing and synergistic effects on tumor cells. Theranostics. 2018;8(7):1824-1849. doi:10.7150/thno.22172

11. Her S, Jaffray DA, Allen C. Gold nanoparticles for applications in cancer radiotherapy: mechanisms and recent advancements. $A d v$ Drug Deliv Rev. 2017;109:84-101. doi:10.1016/j.addr.2015.12.012

12. Herold DM, Das IJ, Stobbe CC, Iyer RV, Chapman JD. Gold microspheres: a selective technique for producing biologically effective dose enhancement. Int $J$ Radiat Biol. 2000;76(10):1357-1364. doi: $10.1080 / 09553000050151637$

13. Hainfeld JF, Slatkin DN, Smilowitz HM. The use of gold nanoparticles to enhance radiotherapy in mice. Phys Med Biol. 2004;49 (18):309-315. doi:10.1088/0031-9155/49/18/N03

14. Kaur H, Pujari G, Semwal MK, Sarma A, Avasthi DK. In vitro studies on radiosensitization effect of glucose capped gold nanoparticles in photon and ion irradiation of HeLa cells. Nucl Instruments Methods Phys Res Sect B Beam Interact with Mater Atoms. 2013;301:7-11. doi:10.1016/j.nimb.2013.02.015

15. Liu Y, Liu X, Jin X, et al. The dependence of radiation enhancement effect on the concentration of gold nanoparticles exposed to low- and high-LET radiations. Phys Medica. 2015;31(3):210-218. doi:10.1016/j.ejmp.2015.01.006

16. Liu X, Liu Y, Zhang P, et al. The synergistic radiosensitizing effect of tirapazamine-conjugated gold nanoparticles on human hepatoma HepG2 cells under X-ray irradiation. Int $J$ Nanomedicine. 2016;11:3517-3531. doi:10.2147/IJN.S105348

17. Frens G. Controlled nucleation for the regulation of the particle size in monodisperse gold suspensions. Nat Phys Sci. 1973;241 (105):20-22. doi:10.1038/physci241020a0

18. Kalimuthu P, John SA. Studies on ligand exchange reaction of functionalized mercaptothiadiazole compounds onto citrate capped gold nanoparticles. Mater Chem Phys. 2010;122(2-3):380-385. doi:10.1016/j.matchemphys.2010.03.009

19. Cheng NN, Starkewolf Z, Davidson RA, et al. Chemical enhancement by nanomaterials under X-ray irradiation. $J$ Am Chem Soc. 2012;134(4):1950-1953. doi:10.1021/ja210239k
20. Tomayko MM, Reynolds CP. Determination of subcutaneous tumor size in athymic (nude) mice. Cancer Chemother Pharmacol. 1989;24 (3):148-154. doi:10.1007/BF00300234

21. Fratoddi I, Venditti I, Battocchio C, et al. Highly hydrophilic gold nanoparticles as carrier for anticancer copper(I) complexes: loading and release studies for biomedical applications. Nanomaterials. 2019;9(5):772. doi:10.3390/nano9050772

22. Ghosh SK. Spectroscopic evaluation of 4-(dimethylamino)pyridine versus citrate as stabilizing ligand for gold nanoparticles. Colloids Surfaces a Physicochem Eng Asp. 2010;371(1-3):98-103. doi:10.1016/j.colsurfa.2010.09.010

23. Hainfeld JF, Lin L, Slatkin DN, Avraham Dilmanian F, Vadas TM, Smilowitz HM. Gold nanoparticle hyperthermia reduces radiotherapy dose. Nanomedicine Nanotechnology, Biol Med. 2014;10 (8):1609-1617. doi:10.1016/j.nano.2014.05.006

24. Bai X, Zhang J, Chang YN, et al. Nanoparticles with high-surface negative-charge density disturb the metabolism of low-density lipoprotein in cells. Int J Mol Sci. 2018;19(9):2790. doi:10.3390/ijms19092790

25. Horiot JC, Begg AC, Le Fur R, et al. Present status of EORTC trials of hyperfractionated and accelerated radiotherapy on head and neck carcinoma. In: Recent Results in Cancer Research. Fortschritte Der Krebsforschung. Progrès Dans Les Recherches Sur Le Cancer. Vol. 134. Springer; 1994:111-119. doi:10.1007/978-3-642-84971-8_13

26. Stuschke M, Thames HD. Hyperfractionated radiotherapy of human tumors: overview of the randomized clinical trials. Int J Radiat Oncol Biol Phys. 1997;37(2):259-267. doi:10.1016/S0360-3016(96)00511-1

27. Eriksson D, Stigbrand T. Radiation-induced cell death mechanisms. Tumor Biol. 2010;31(4):363-372. doi:10.1007/s13277-010-0042-8

28. Belka C, Jendrossek V, Pruschy M, Vink S, Verheij M, Budach W. Apoptosis-modulating agents in combination with radiotherapy Current status and outlook. Int J Radiat Oncol Biol Phys. 2004;58 (2):542-554. doi:10.1016/j.jirobp.2003.09.067

29. Kubo N, Noda SE, Takahashi A, et al. Radiosensitizing effect of carboplatin and paclitaxel to carbon-ion beam irradiation in the non-small-cell lung cancer cell line H460. J Radiat Res. 2015;56 (2):229-238. doi:10.1093/jrr/rru085

30. Boag JW. Twelfth Failla Memorial Lecture the Time Scale in Radiobiology; Academic Press; 1975. doi:10.1016/b978-0-12523350-7.50009-2

31. Rudek B, McNamara A, Ramos-Méndez J, Byrne H, Kuncic Z, Schuemann J. Radio-enhancement by gold nanoparticles and their impact on water radiolysis for $\mathrm{x}$-ray, proton and carbon-ion beams. Phys Med Biol. 2019;64(17):175005. doi:10.1088/1361-6560/ab314c

32. Fuss MC, Boscolo D, Durante M, Scifoni E, Kramer M. Systematic quantification of nanoscopic dose enhancement of gold nanoparticles in ion beams. Phys Med Biol. 2020;65(7):75008. doi:10.1088/1361-6560/ ab7504

33. Lesueur P, Chevalier F, El-Habr EA, et al. Radiosensitization Effect of Talazoparib, a Parp Inhibitor, on Glioblastoma Stem Cells Exposed to Low and High Linear Energy Transfer Radiation. Sci Rep. 2018;8 (1):3664. doi:10.1038/s41598-018-22022-4

34. Matsumoto K, Nyui M, Ueno M, Ogawa Y, Nakanishi I. A quantitative analysis of carbon-ion beam-induced reactive oxygen species and redox reactions. J Clin Biochem Nutr. 2019;65(4):1-7. doi:10.3164/jcbn.18-34

35. Roots R, Okada S. Protection of DNA molecules of cultured mammalian cells from radiation-induced single-Strand scissions by various alcohols and SH compounds. Int J Radiat Biol. 1972;21 (4):329-342. doi:10.1080/09553007214550401

36. Hirayama R, Ito A, Tomita $\mathrm{M}$, et al. Contributions of direct and indirect actions in cell killing by high-LET radiations. Radiat Res. 2009;171(2):212-218. doi:10.1667/RR1490.1

37. Aranda A, Sequedo L, Tolosa L, et al. Dichloro-dihydro-fluorescein diacetate (DCFH-DA) assay: a quantitative method for oxidative stress assessment of nanoparticle-treated cells. Toxicol Vitr. 2013;27 (2):954-963. doi:10.1016/j.tiv.2013.01.016 
38. Cui T, Li S, Chen S, Liang Y, Sun H, Wang L. Stealth" dendrimers with encapsulation of indocyanine green for photothermal and photodynamic therapy of cancer. Int J Pharm. 2021;600. doi:10.1016/j. ijpharm.2021.120502.

39. Wolff CM, Kolb JF, Weltmann KD, von Woedtke T, Bekeschus S. Combination treatment with cold physical plasma and pulsed electric fields augments ROS production and cytotoxicity in lymphoma. Cancers (Basel). 2020;12:4. doi:10.3390/cancers12040845

40. Schlinkert P, Casals E, Boyles M, et al. The oxidative potential of differently charged silver and gold nanoparticles on three human lung epithelial cell types. $J$ Nanobiotechnology. 2015;13(1):1. doi:10.1186/s12951-014-0062-4

41. Vandersickel V, Depuydt J, van Bockstaele B, et al. Early increase of radiation-induced $\gamma \mathrm{H} 2 \mathrm{AX}$ foci in a human ku70/80 knockdown cell line characterized by an enhanced radiosensitivity. $J$ Radiat Res. 2010;51(6):633-641. doi:10.1269/jrr.10033

42. Antonsson B, Martinou JC. The Bcl-2 protein family. Exp Cell Res. 2000;256(1):50-57. doi:10.1006/excr.2000.4839

43. Liu X, Sun C, Jin X, et al. Genistein enhances the radiosensitivity of breast cancer cells via G2/M cell cycle arrest and apoptosis. Molecules. 2013;18(11):13200-13217. doi:10.3390/molecules 18 1113200

44. Cohen GM. Caspases: the executioners of apoptosis. Biochem J. 1997;326(1):1-16. doi:10.1042/bj3260001

45. Liou AKF, Clark RS, Henshall DC, Yin XM, Chen J. To die or not to die for neurons in ischemia, traumatic brain injury and epilepsy: a review on the stress-activated signaling pathways and apoptotic pathways. Prog Neurobiol. 2003;69(2):103-142. doi:10.1016/ S0301-0082(03)00005-4
46. Nie C, Luo Y, Zhao X, et al. Caspase-9 mediates PUMA activation in UCN-01-induced apoptosis. Cell Death Dis. 2014;5(10):e1495. doi:10.1038/cddis.2014.461

47. Strappazzon F, Torch S, Chatellard-Causse C, et al. Alix is involved in caspase 9 activation during calcium-induced apoptosis. Biochem Biophys Res Commun. 2010;397(1):64-69. doi:10.1016/j. bbrc.2010.05.062

48. Brentnall M, Rodriguez-Menocal L, De Guevara RL, Cepero E, Boise LH. Caspase-9, caspase-3 and caspase-7 have distinct roles during intrinsic apoptosis. BMC Cell Biol. 2013;14(1):32. doi:10.1186/1471-2121-14-32

49. Subiel A, Ashmore R, Schettino G. Standards and methodologies for characterizing radiobiological impact of high- $Z$ nanoparticles. Theranostics. 2016;6(10):1651-1671. doi:10.7150/THNO.15019

50. Chang MY, Shiau AL, Chen YH, Chang CJ, Chen HHW, Wu CL. Increased apoptotic potential and dose-enhancing effect of gold nanoparticles in combination with single-dose clinical electron beams on tumor-bearing mice. Cancer Sci. 2008;99(7):1479-1484. doi:10.1111/j.1349-7006.2008.00827.x

51. Mousavie Anijdan SH, Mahdavi SR, Shirazi A, Zarrinfard MA, Hajati J. Megavoltage X-ray dose enhancement with gold nanoparticles in tumor bearing mice. Int $J$ Mol Cell Med. 2013;2(3):118-123.

52. Komatsu T, Nakamura K, Okumura Y, Konishi K. Optimal method of gold nanoparticle administration in melanoma-bearing mice. Exp Ther Med. 2018;15(3):2994-2999. doi:10.3892/etm.2018.5746
International Journal of Nanomedicine

\section{Publish your work in this journal}

The International Journal of Nanomedicine is an international, peerreviewed journal focusing on the application of nanotechnology in diagnostics, therapeutics, and drug delivery systems throughout the biomedical field. This journal is indexed on PubMed Central, MedLine, CAS, SciSearch ${ }^{\mathbb{B}}$, Current Contents ${ }^{\mathbb{B}} /$ Clinical Medicine, $^{2}$

\section{Dovepress}

Journal Citation Reports/Science Edition, EMBase, Scopus and the Elsevier Bibliographic databases. The manuscript management system is completely online and includes a very quick and fair peer-review system, which is all easy to use. Visit http://www.dovepress.com/ testimonials.php to read real quotes from published authors. 